\title{
K-THEORY OF STABLE GENERALIZED OPERATOR ALGEBRAS
}

\author{
Hvedri Inassaridze \\ A.Razmadze Mathematical Institute, Georgian Academy of Sciences, \\ M.Alexidze St. 1, Tbilisi 380093. Georgia \\ hvedri@rmi.acnet.ge \\ Tamaz Kandelaki \\ A.Razmadze Mathematical Institute, Georgian Academy of Sciences, \\ M.Alexidze St. 1, Tbilisi 380093. Georgia \\ kandel@rmi.acnet.ge
}

\begin{abstract}
It is proved that algebraic and topological $K$-functors are isomorphic on the category of stable generalized operator algebras which are $K_{i}$-regular for all $i>0$.
\end{abstract}

In 1979 Karoubi conjectured the isomorphism of algebraic and topological $K$ functors on the category of stable $C^{*}$-algebras $[\mathrm{Ka}]$. This conjecture was confirmed by Higson for Karoubi-Villamayor algebraic $K$-functors [Hi] and by Suslin and Wodzicki for Quillen algebraic $K$-functors [SW]. Further it was proved by the first author [In1] that algebraic and topological $K$-functors are isomorphic for polynomial extensions of stable $C^{*}$-algebras.

Our aim is to confirm Karoubi's conjecture for a wider class of complex topological algebras. Namely it is proved the equality of algebraic and topological $K$-functors on the category of stable generalized operator algebras (Theorems 9 and 11).

Generalized operator algebras are projective limits of $C^{*}$-algebras in the category of complex involutive algebras equipped with Hausdorff topology such that all algebraic operations are continuous. In our paper generalized operator algebras will be called shortly pro- $C^{*}$-algebras. Such algebras have been introduced and studied by Arveson [Ar] for countable inverse systems of $C^{*}$-algebras and by Weidner [We] for arbitrary inverse systems of $C^{*}$-algebras.

We begin by the formulation of sufficient conditions establishing the relationship between algebraic and topological $K$-functors on the category of real (or complex) topological algebras.

Let $A$ be a real topological algebra with unit. $K_{n}(A)$ denotes the Quillen algebraic $K$-functor for $n \geq 0$ and the negative algebraic $K$-functor for $n<0, \kappa_{n}(A), n>0$, denotes the Karoubi-Villamayor algebraic $K$-functor.

2000 Mathematics Subject Classification. 19D06, 19D25, 46L80, 46M15, 55P65, 55R45.

Key words and phrases. $K$-functor, stable pro- $C^{*}$-algebra, homotopy functor, monoid algebra.

Partially supported by INTAS Georgia grant No 213. The first author was also partially supported by NATO linkage grant No 975316. 
$K_{n}^{\text {top }}(A)=\pi_{n-1}\left(G L(A)^{\text {top }}\right)$ denotes the topological $K$-functor for $n>0$, where $G L(A)^{\text {top }}$ is the topological linear group equipped with the inductive limit topology induced by the topology of $A$ and $K_{0}^{\text {top }}$ coincides with the Grothendieck functor $K_{0}$.

One has natural maps

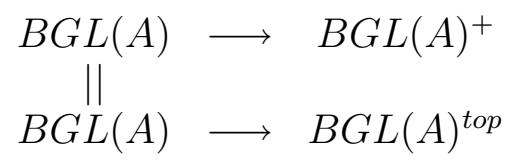

and by the universal property of Quillen plus construction one gets a continuous map

$$
\varphi_{A}: B G L(A)^{+} \longrightarrow B G L(A)^{\text {top }}
$$

making the aforegiven diagram commutative up to homotopy. Therefore $\varphi_{A}$ induces a natural homomorphism $\varphi_{A}: K_{n}(A)=\pi_{n}\left(B G L(A)^{+}\right) \rightarrow \pi_{n}\left(B G L(A)^{\text {top }}\right)=$ $K_{n}^{\text {top }}(A)$ for all $n>0$. The definition of $\varphi_{*}$ is extended to the non unital case in the usual way.

Let $\mathcal{P}=(P, \tau, \delta)$ and $\mathcal{J}=(J, \tau, \delta)$ be the polynomial cotriple and the path cotriple respectively in the category of topological $\mathbb{R}$-algebras. Then by definition

$$
\kappa_{n}(A)=\pi_{n-1} G L\left(P_{*}(A)\right), \quad K_{n}^{\mathrm{top}}(A)=\pi_{n-1} G L\left(J_{*}(A)\right)
$$

for $n>2$ and one has the following exact sequences

$$
\begin{gathered}
0 \longrightarrow \kappa_{2}(A) \longrightarrow \pi_{0} G L\left(P_{*}(A)\right) \longrightarrow G L(A) \longrightarrow \kappa_{1}(A) \longrightarrow 0, \\
0 \longrightarrow K_{2}^{\text {top }}(A) \longrightarrow \pi_{0} G L\left(J_{*}(A)\right) \longrightarrow G L(A) \longrightarrow K_{1}^{\text {top }}(A) \longrightarrow 0 .
\end{gathered}
$$

The natural morphism of cotriples $\eta: \mathcal{P} \rightarrow \mathcal{J}$ given by

$$
\eta_{A}\left(\sum_{i=1}^{k} a_{i} x^{i}\right)=\sum_{i=1}^{k} a_{i} t^{i}, \quad t \in I=[0 ; 1], \quad a_{i} \in A
$$

induces a canonical homomorphism $\beta_{n}: \kappa_{n}(A) \rightarrow K_{n}^{\text {top }}(A)$ for all $n>0$.

As noted before the homomorphisms $\varphi_{n}$ and $\beta_{n}, n>0$, are isomorphisms if $A$ is a stable $C^{*}$-algebra.

Theorem A. Let $A$ be a topological $\mathbb{R}$-algebra (not necessarily with unit).

1) If $i$ is a non negative integer then there is an isomorphism

$$
\varphi_{i+1}: K_{i+1}(A) \longrightarrow K_{i+1}^{\mathrm{top}}(A)
$$

if $K_{i+1}(A)=K_{i+1}\left(A^{I}\right)$ and $K_{j+1}\left(B^{I^{i-j-1}}\right)=K_{j+1}\left(B^{I^{i-j+1}}\right)$ for all $0 \leq j \leq i-1$, where $A^{I}$ is the path algebra of $A$ and $B=A \times \cdots \times A$ is the $2^{j}$ times product of $A$ on itself.

2) If $A$ is with unit then one has an isomorphism

$$
\varphi_{n}: K_{n}(A) \longrightarrow K_{n}^{\mathrm{top}}(A)
$$

for all $n>0$, if $K_{i}(A)=K_{i}\left(A^{I^{m}}\right)$ for all $i, m>0$.

Theorem B. For any topological $\mathbb{R}$-algebra the homomorphism $\beta_{*}: \kappa_{*}(A) \rightarrow$ $K_{*}^{\mathrm{top}}(A)$ is an isomorphism if $\kappa_{*}(A)=\kappa_{*}\left(A^{I^{m}}\right)$ for all $m>0$. 
Theorems A and B have been proved in detail for normed $\mathbb{R}$-algebras in [In1], Theorems 10 and 22 . The proof for topological $\mathbb{R}$-algebras is completely similar and will be omited.

We see that conditions of these Theorems are the topological analogue of $K_{*^{-}}$ regularity of discrete rings, implying the isomorphism of Quillen and KaroubiVillamayor algebraic $K$-functors, polynomial rings replaced by path algebras.

We see also for the equality of algebraic and topological $K$-functors on a subcategory of the category of real (or complex) topological algebras it is necessary to prove that the algebraic $K$-functors are homotopy functors on this subcategory. To this end a key role plays the triple factorization property of discrete rings introduced by Suslin and Wodzicki [SW]. We recall the definition.

It is said that a ring $A$ possesses the property $(T F)_{\text {right }}$ if for any collection $a_{1}, \ldots, a_{m} \in A$ there exist $b_{1}, \ldots, b_{m}, c, d \in A$ such that $a_{i}=b_{i} c d$ for $1 \leq i \leq m$ and the left annihilators in $A$ of $c$ and $c d$ are equal.

The rest of the paper is devoted to the particular case of generalized operator algebras showing that they satisfy conditions of Theorems $A$ and $B$.

Let $\underline{\mathcal{A}}$ be the category of involutive complex algebras equipped with Hausdorff topology such that all algebraic operations are continuous. Morphisms of this category are involutive continuous algebra homomorphisms.

An algebra of the category $\underline{\mathcal{A}}$ is called a generalized operator algebra if there exists an inverse system $\left\{A_{\alpha}\right\}$ of $C^{*}$-algebras $A_{\alpha}$ such that

$$
\underset{\lim }{\longleftarrow}\left\{A_{\alpha}\right\}=A
$$

(see $[\mathrm{We}])$.

For a complex algebra $A$ in $\underline{\mathcal{A}}$ let $S(A)$ be the set of continuous $C^{*}$-seminorms. For each $\alpha \in S(A)$ let $I_{\alpha}=\left\{a \in A:|a|_{\alpha}=0\right\}$ and $A_{\alpha}=A / I_{\alpha}$ which is a $C^{*}$-algebra. Then one gets an inverse system of $C^{*}$-algebras $\left\{A_{\alpha}, \pi^{\beta \alpha}: A_{\beta} \longrightarrow A_{\alpha}, \beta \geq \alpha\right\}$ and let $\pi^{\alpha}: A \longrightarrow A_{\alpha}, \alpha \in S(A)$, be canonical projections which induce a morphism

$$
\tau: A \longrightarrow A_{\infty}=\longleftarrow \lim _{\longleftarrow}\left\{A_{\alpha}, \pi^{\beta \alpha}\right\} .
$$

In [We] the following characterization of generalized operator algebras is given.

Proposition 1. (i) $A$ is a generalized operator algebra,

(ii) $A$ is complete and the topology on $A$ is induced by a system of $C^{*}$-seminorms,

(iii) the canonical homomorphism $\pi: A \rightarrow A_{\infty}$ is an isomorphism of involutive algebras and a homeomorphism.

Such algebras will be called pro- $C^{*}$-algebras. The full subcategory of $\underline{\mathcal{A}}$ consisting of all pro- $C^{*}$-algebras will be denoted by $\operatorname{Pr} C^{*}$.

An element $a$ of a pro- $C^{*}$-algebra $A$ is called bounded if $\|a\|_{b}=\sup _{\alpha}\left\{|a|_{\alpha}\right\}$ is finite. The subalgebra of bounded elements of $A$ is a $C^{*}$-algebra with respect to this norm and the canonical injection $\tau: A_{b} \rightarrow A$ is continuous and dense [We].

Proposition 2. Let $A$ be a pro- $C^{*}$-algebra and $A_{b}$ be the $C^{*}$-algebra of bounded elements of $A$. Then 
(i) the canonical $*$-homomorphism $\tau: A_{b} \rightarrow A$ induces an isomorphism

$$
\tau_{0}: K_{0}\left(A_{b}\right) \longrightarrow K_{0}(A),
$$

(ii) the functor $\mathcal{L}: \underline{\operatorname{Pr} C^{*}} \rightarrow \underline{C^{*}}$ (the category of $C^{*}$-algebras), given by $\mathcal{L}(A)=A_{b}$, is the right adjoint to the canonical inclusion $\mathcal{I}: \underline{C^{*}} \rightarrow \underline{\operatorname{Pr} C^{*}}$, i.e. there is a natural bijection

$$
\lambda: \operatorname{Hom}_{\underline{\operatorname{Pr} C^{*}}}(\mathcal{I}(A), B) \approx \operatorname{Hom}_{\underline{C}^{*}}(A, \mathcal{L}(B)) .
$$

Proof. (i) To show that $\tau_{0}$ is surjective it is sufficient to prove that any idempotent is isomorphic to a projection (self-adjoint idempotent) in $A$. Let $p$ be an idempotent of $A$ and $p_{\alpha}$ denote the canonical image of $p$ in the $C^{*}$-algebra $A_{\alpha}$. Since $\left(2 p_{\alpha}-\right.$ 1) $\left(2 p_{\alpha}^{*}-1\right)$ is positive in $A_{\alpha}$, the element $\left(2 p_{\alpha}-1\right)\left(2 p_{\alpha}^{*}-1\right)+1$ is invertible for all $\alpha$ implying that $(2 p-1)\left(2 p^{*}-1\right)+1$ is invertible in $A$. Using functional calculus (see [We]) there follows that the element $\left((2 p-1)\left(2 p^{*}-1\right)+1\right)^{1 / 2}$ is also invertible and the element $q=\left((2 p-1)\left(2 p^{*}-1\right)+1\right)^{1 / 2} p\left((2 p-1)\left(2 p^{*}-1\right)+1\right)^{-1 / 2}$ is a projection. Let $p$ and $q$ be isomorphic projections. Then there is an invertible element $v$ such that $p=v q v^{-1}$. By functional calculus take $u=\left(v^{*} v\right)^{-1 / 2}$ which is an unitary element. It is easily checked that $p=u q u^{*}$. Therefore $p$ and $q$ are unitary isomorphic implying that $\tau_{0}$ is injective.

(ii) Let $A$ be a $C^{*}$-algebra, $B$ be a pro- $C^{*}$-algebra and $f: A \rightarrow B$ be a bounded $*$ homomorphism. Composing $f$ with the canonical bounded homomorphism $B \rightarrow B_{\alpha}$ for each $C^{*}$-seminorm $\alpha$ on $B$ one gets $\|f(a)\|_{\alpha} \leq\|a\|$ for any $a \in A$. Therefore the map given by $\lambda(f)(a)=f(a), a \in A$, is well defined. Conversely, the inverse map is defined by using the composite $A \rightarrow \mathcal{L}(B) \subset B$.

A direct system $\left\{u_{\gamma}\right\}$ of bounded elements of a pro- $C^{*}$-algebra $A$ is called a right bounded approximate unit for $A$ if $a u_{\gamma} \rightarrow a$ for each $a \in A$.

Lemma 3. Any pro-C*-algebra possesses a right bounded approximate unit.

Proof. Let $\left\{u_{\gamma}\right\}$ be a right approximate unit for $A_{b}$. Then $\lim a u_{\gamma}=a$ for each $a \in A$. In effect the canonical homomorphism $\pi^{\alpha} \tau: A_{b} \rightarrow A_{\alpha}$ is surjective and $\left\{v_{\gamma}\right\}=\left\{\pi^{\alpha} \tau\left(u_{\gamma}\right)\right\}$ is a right approximate unit for each $A_{\alpha}$. Thus $\left\|a_{\alpha} v_{\gamma}-a_{\alpha}\right\|_{\alpha} \rightarrow 0$, where $a_{\alpha}=\pi^{\alpha}(a), \alpha \in S(A)$.

We will need the following result which is a part of Proposition 1.8 [We].

Lemma 4. Let $E$ be a Hilbert module over a pro- $C^{*}$-algebra $A$. Then for any $e \in E$ there exist $e_{0} \in E$ and $a \in A$ such that $e=e_{0} a$.

Theorem 5. Any monoid algebra $A[M]$ over a pro- $C^{*}$-algebra $A$ has the right factorization property $(T F)_{\text {right }}$.

Proof. At first the assertion will be shown for $A$. Let $e=\left(a_{1}, \ldots, a_{m}\right)$ be an element of the standard Hilbert $A$-module $A^{m}=A \oplus \cdots \oplus A$. Then by Lemma 4 there exist $e_{0}=\left(b_{1}, \cdots, b_{m}\right) \in A^{m}$ and $a \in A$ such that $e=e_{0} a$. The closure $\overline{a A}$ in $A$ of $a A$ contains the element $a$, since by Lemma 3 the pro- $C^{*}$-algebra $A$ has a right bounded approximate unit. Applying again Lemma 4 to the Hilbert submodule $\overline{a A}$ one gets a factorization $a=c d$ for some $c \in \overline{a A}$ and $d \in A$. Therefore if $r a=0$, then $r \cdot \overline{a A}=0$. There follows that the left annihilators in $A$ of $c$ and $c d$ are equal. 
The $(T F)_{\text {right }}$ property for $A[M]$ follows now from the general fact claiming that if a ring $A$ has the $(T F)_{\text {right }}$ property then any monoid algebra $A[M]$ over $A$ has the $(T F)_{\text {right }}$ property too. This assertion is proved similarly to the particular case of polynomial extensions of $C^{*}$-algebras (See [In1], Lemma 16) and is omited.

Corollary 6. If $A$ is a pro- $C^{*}$-algebra then any monoid algebra $A[M]$ over $A$ satisfy excision and is Morita invariant in algebraic $K$-theory.

Therefore if $R$ is a ring with unit containing $A[M]$ as a two-sided ideal, then the canonical map $K_{*}(A[M]) \rightarrow K_{*}(R, A[M])$ is an isomorphism and the embedding $A[M] \rightarrow M_{k}(A[M])$ induces an isomorphism $K_{*}(A[M]) \rightarrow K_{*}\left(M_{k}(A[M])\right)$.

Corollary 6 follows immediately from Theorem 5 by Theorem 3.11, Corollary 1.20 and Corollary 3.12 of [SW].

Let $A$ be a pro- $C^{*}$-algebra and $B$ be a $C^{*}$-algebra. Then the tensor product $A \hat{\otimes} B$ is defined as the projective limit in $\mathcal{A}$ of the inverse system

$$
\left\{A_{\alpha} \hat{\otimes} B, \pi^{\beta \alpha} \hat{\otimes} 1_{B}, \quad \alpha, \beta \in S(A)\right\}
$$

(see $[\mathrm{We}])$.

Definition 7. A pro- $C^{*}$-algebra $A$ is called stable if it has the form $B \hat{\otimes} \mathcal{K}$ for some pro- $C^{*}$-algebra $B$, where $\mathcal{K}$ is the $C^{*}$-algebra of compact operators on the infinite dimensional Hilbert space $\mathcal{H}$.

Clearly a stable pro- $C^{*}$-algebra is the projective limit of stable $C^{*}$-algebras.

Now Higson homotopy invariance theorem [Hi] will be extended to the category $\underline{\operatorname{Pr} C^{*}}$ of pro- $C^{*}$-algebras.

Homomorphisms $f_{0}, f_{1}: A \rightarrow A^{\prime}$ of pro- $C^{*}$-algebras are said to be homotopic $\left(f_{0} \sim f_{1}\right)$ if there exists a homomorphism $F: A \rightarrow A^{\prime} \hat{\otimes} \mathbb{C}^{I}$ such that $\left(1 \hat{\otimes} \varepsilon_{0}\right) F=$ $f_{0} \hat{\otimes} 1$ and $\left(1 \hat{\otimes} \varepsilon_{1}\right) F=f_{1} \hat{\otimes} 1$, where $\varepsilon_{0}, \varepsilon_{1}: \mathbb{C}^{I} \rightarrow \mathbb{C}$ are given by $\varepsilon_{0}(g)=g(0)$ and $\varepsilon_{1}(g)=g(1)$ for $g: I=[0 ; 1] \rightarrow \mathbb{C}$. Note that $A \hat{\otimes} \mathbb{C}^{I}$ is naturally isomorphic to $A^{I}$ [We].

Let $T: \underline{\operatorname{Pr} C^{*}} \rightarrow \underline{A b}$ be a functor to the category of abelian groups. It is said that

(a) $T$ is a homotopy functor if $T\left(f_{0}\right)=T\left(f_{1}\right)$ for $f_{0} \sim f_{1}$.

(b) $T$ is stable if $T\left(1_{A} \hat{\otimes} e\right)$ is an isomorphism, where $e: \mathbb{C} \rightarrow \mathcal{K}$ is the orthogonal projection on the first basis vector of the infinite dimensional Hilbert space $\mathcal{H}$.

(c) $T$ is split exact if for any split extension

$$
0 \longrightarrow A_{1} \longrightarrow A \longrightarrow A_{2} \longrightarrow 0
$$

of pro- $C^{*}$-algebras the induced sequence

$$
0 \longrightarrow T\left(A_{1}\right) \longrightarrow T(A) \longrightarrow T\left(A_{2}\right) \longrightarrow 0
$$

is split exact in the category $\underline{A b}$.

It is easily checked that $T$ is a homotopy functor if and only if the inclusion $i: A \rightarrow A^{I}$ induces an isomorphism $T(i): T(A) \rightarrow T\left(A^{\prime}\right)$ for any pro- $C^{*}$-algebra $A$.

Theorem 8. Let $T: \underline{\operatorname{Pr} C^{*}} \rightarrow \underline{A b}$ be a stable and split exact functor. Then $T$ is a homotopy functor. 
Proof. Let $\underline{C_{\text {nucl }}^{*}}$ be the category of separable nuclear $C^{*}$-algebras. The functor $T_{B}: C_{\text {nucl }}^{*} \rightarrow \underline{A b}$ defined by $T_{B}(A)=T(B \hat{\otimes} A)$ and $T_{B}(g)=T\left(1_{B} \hat{\otimes} g\right)$ is stable and split exact. According to Higson homotopy invariance theorem one gets that for any $B \in \underline{\operatorname{Pr} C^{*}}$ the functor $T_{B}$ is a homotopy functor. Therefore the homomorphisms $T\left(1_{B} \hat{\otimes} \varepsilon_{0}\right), T\left(1_{B} \hat{\otimes} \varepsilon_{1}\right)$ are equal for any pro- $C^{*}$-algebra implying the homotopy property of the functor $T$.

Clearly the Grothendieck functor $K_{0}$ is a homotopy functor on the subcategory of stable pro- $C^{*}$-algebras and the topological $K$-functors $K_{n}^{\text {top }}, n>0$, are homotopy functors on the category of real topological algebras.

Theorem 9. Let $A$ be a stable pro- $C^{*}$-algebra. Then

(1) the natural homomorphism $\varphi_{n}: K_{n}(A) \rightarrow K_{n}^{\mathrm{top}}(A)$ is an isomorphism for all $n \geq 1$,

(2) the natural homomorphism $\beta_{n}: \kappa_{n}(A) \longrightarrow K_{n}^{\text {top }}(A)$ is an isomorphism for all $n \geq 1$.

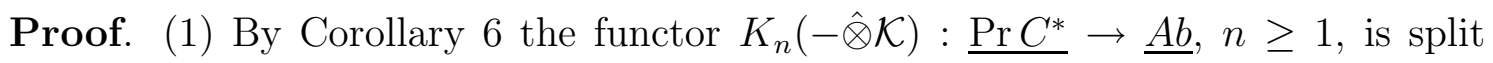
exact and it is standard using Morita invariance property (see [SW], [In1]) to show the stability of the functor $K_{n}(-\hat{\otimes} \mathcal{K}), n \geq 1$. By Theorem 8 these functors are homotopy functors and it remains to apply Theorem A.

(2) The functor $\kappa_{n}(-\hat{\otimes} \mathcal{K}): \underline{\operatorname{Pr} C^{*}} \rightarrow A b, n \geq 1$, is a homotopy functor. In effect, it is well known that Karoubi-Villamayor algebraic $K$-functors $\kappa_{n}$ preserve split exactness. To show the stability of the functor $\kappa_{n}(-\hat{\otimes} \mathcal{K})$ it is necessary to prove that for any ring $A$ the natural inclusion $A \rightarrow M_{2}(A)$ induces an isomorphism $\kappa_{n}(A) \approx \kappa_{n}\left(M_{2}(A)\right), n \geq 1$. Since $\kappa_{n+1}(A)=\kappa_{n}(\Omega A), n \geq 1$, where $\Omega A$ is the kernel of $\delta: t A[t] \rightarrow A, \delta\left(\sum a_{i} t^{i}\right)=\sum a_{i}$ and $\Omega\left(M_{k}(A)\right)=M_{k}(\Omega A)$, it is sufficient to prove the isomorphism $\kappa_{1}(A) \rightarrow \kappa_{1}\left(M_{2}(A)\right)$. But this is done in [Hi]. Therefore the functor $\kappa_{n}(-\hat{\otimes} \mathcal{K})$ is a homotopy functor for all $n \geq 1$. Apply now theorem $\mathrm{B}$ to get the required isomorphism of $K$-functors.

We recall that for a locally compact space $X$ the algebra $C_{0}(X)$ denotes the algebra of continuous complex valued functions on $X$ tending to zero at infinity.

Theorem 10. Let $A$ be a stable pro-C*-algebra. Then one has the canonical Bott isomorphism

$$
K_{n}\left(\Omega^{2} A\right) \approx K_{n}(A)
$$

for all $n \in Z$, where $\Omega^{2}(A)=A \hat{\otimes} C_{0}(0 ; 1) \hat{\otimes} C_{0}(0 ; 1)=A \hat{\otimes} C_{0}(\mathbb{C})$.

Proof. The definition of negative algebraic $K$-functors $\left(K_{-n}(A)=K_{0}\left(s^{n} A\right), n \geq 1\right.$, where $s A$ is the suspension of $A$ ) implies that they have the exactness and Morita invariance properties. We deduce that the functors $K_{n}(A \hat{\otimes}-): C_{\text {nucl }}^{*} \rightarrow A b$ are homotopy functors for all $n \in Z$. Use now the following weak form of Cuntz periodicity theorem $[\mathrm{Cu}]$ :

If $E$ is stable, half exact and homotopy functor from the category $C_{\text {nucl }}^{*}$ of separable nuclear $C^{*}$-algebras to the category of abelian groups, then there is a canonical isomorphism $E\left(C_{0}\left(\mathbb{R}^{2}\right)\right) \approx E(\mathbb{C})$. 
Clearly the functors $K_{n}(A \hat{\otimes}-), n \in Z$, satisfy conditions of Cuntz periodicity theorem and one gets the required Bott periodicity.

Let $A[M]$ be a monoid algebra over a pro- $C^{*}$-algebra $A$. Then in a natural way $A[M]$ is the inductive limit of the $A$-submodules $A\left[M_{\alpha}\right]$, where the limit is taken over all finite subsets $M_{\alpha}$ of the monoid $M$. The topology of $A$ induces on each $A\left[M_{\alpha}\right]$ the product topology and $A[M]$ becomes a complex topological algebra with respect to the inductive topology.

A complex topological algebra $B$ is called a retract of a monoid algebra $A[M]$ over a pro- $C^{*}$-algebra $A$ if there exist homomorphisms of complex topological algebras $f: B \rightarrow A[M], g: A[M] \rightarrow B$ such that $g f=1_{B}$.

Theorem 11. If $B$ is a retract of a monoid algebra $A[M]$ over a stable pro- $C^{*}$ algebra $A$ then one has isomorphisms

$$
\varphi_{n}: K_{n}(B) \longrightarrow K_{n}^{\mathrm{top}}(B), \beta_{n}: \kappa_{n}(B) \longrightarrow K_{n}^{\mathrm{top}}(B), \quad n \geq 1
$$

Proof. At first consider the case $B=A[M]$ and the functors $K_{n}((-\hat{\otimes} \mathcal{K})[M])$, $\kappa_{n}((-\hat{\otimes} \mathcal{K})[M]): \underline{\operatorname{Pr} C^{*}} \rightarrow \underline{A b}, n \geq 1$. It will be shown that they are homotopy functors. In effect, they are split exact, since tensoring by $\mathcal{K}$, then taking the monoid algebra preserve split exactness and use Corollary 6 for the case $K_{*}$. The stability follows from the Morita invariance property and the equality $M_{k}(A[M])=$ $M_{k}(A)[M]$ for any $A$. Therefore by Theorem 8 the functors $K_{n}((-\hat{\otimes} \mathcal{K})[M])$ and $\kappa_{n}((-\hat{\otimes} \mathcal{K})[M]), n \geq 1$, are homotopy functors. Thus the inclusion $A \rightarrow A^{I}$ induces an isomorphism

$$
K_{*}((A \hat{\otimes} \mathcal{K})[M]) \approx K_{*}\left(\left(A^{I} \hat{\otimes} \mathcal{K}\right)[M]\right)=K_{*}\left((A \hat{\otimes} \mathcal{K})[M]^{I}\right),
$$

and

$$
K_{*}((A \hat{\otimes} \mathcal{K})[M]) \times((A \hat{\otimes} \mathcal{K})[M])=K_{*}\left((A \hat{\otimes} \mathcal{K}[M]) \times K_{*}((A \hat{\otimes} \mathcal{K})[M]) .\right.
$$

The same is true for $\kappa_{*}((-\hat{\otimes} \mathcal{K})[M])$.

All conditions of Theorem A and B respectively are satisfied implying the required isomorphism of algebraic and topological $K$-functors for monoid algebras over stable pro- $C^{*}$-algebras.

If $B$ is a retract of $A[M]$ then one has isomorphisms $K_{*}(B) \approx K_{*}\left(B^{I^{m}}\right), \kappa_{*}(B) \approx$ $\kappa_{*}\left(B^{I^{m}}\right), m>0$, induced by the inclusion $B \rightarrow B^{I^{m}}$, and $K_{*}\left(B^{I^{m}} \times \cdots \times B^{I^{m}}\right) \approx$ $K_{*}\left(B^{I^{m}}\right) \times \cdots \times K_{*}\left(B^{I^{m}}\right), m \geq 0$. For $B$ apply again Theorems $\mathrm{A}$ and $\mathrm{B}$ respectively.

Theorem 11 is proved in [In1], Theorem 18, Remark 19 and Proposition 21, for particular cases of the monoid $M$ when $A$ is a stable $C^{*}$-algebra.

Corollary 12. Any stable pro- $C^{*}$-algebra is $K_{i}$-regular for all $i \geq 1$.

Proof. Denote $\alpha_{n}: K_{n}(A) \rightarrow \kappa_{n}(A), n \geq 1$, the well known homomorphism between Quillen and Karoubi-Villamayor algebraic $K$-functors [AKW], [In2] for any $\operatorname{ring} A$. Let $A$ be a stable pro- $C^{*}$-algebra. The inclusion $A \rightarrow A\left[x_{1}, \ldots, x_{k}\right]$ induces 
a commutative diagram

$$
\begin{array}{ccc}
K_{n}(A) & \longrightarrow & K_{n}\left(A\left[x_{1}, \ldots, x_{k}\right]\right) \\
\alpha_{n} \downarrow & & \downarrow \alpha_{n} \\
\kappa_{n}(A) & \longrightarrow & \kappa_{n}\left(A\left[x_{1}, \ldots, x_{k}\right]\right)
\end{array}
$$

where the bottom map is an isomorphism. Since $\beta_{n} \alpha_{n}=\varphi_{n}, n \geq 1$, by Theorem 11 the vertical maps of this diagram are also isomorphisms, implying the isomorphism of the top map for all $n \geq 1$ and $k \geq 1$.

For the case of $C^{*}$-algebras the $K$-regularity has been investigated by Rosenberg [Ro] and by the first author [In1].

\section{REFERENCES}

[AKW] D.W. Anderson, M. Karoubi and J. Wagoner, Relations between higher algebraic Ktheories, Algebraic K-theory I, Lecture Notes in Math. 341, pp. 68-76, Springer-Verlag, 1973.

[Ar] W.Arveson, The harmonic analysis of automorphism groups, Operator Algebras and Applications, part 1, Proc. of Symp. in Pure Math. 38 (1982), 199-269.

[Cu] J.Cuntz, K-theory and $C^{*}$-algebras, Lecture Notes in Math. 1046, Springer-Verlag, (1983), 55-79.

[Hi] N.Higson, Algebraic K-theory of stable $C^{*}$-algebras, Adv. Math. 67 (1988), 1-140.

[In1] H.Inassaridze, Algebraic K-theory of normed algebras, $K$-Theory 21, No 1 (2000), 25-56.

[In2] H.Inassaridze, Algebraic K-theory, 440 pages, Kluwer Academic Publishers, 1995.

[Ka] M.Karoubi, $K$-théorie algébrique de certaines algébres d'opérateurs, Lecture Notes in Math. 725, Springer-Verlag, (1979), 254-290.

[Ro] J.Rosenberg, The algebraic $K$-theory of operator algebras, $K$-Theory 12, No 1 (1997), 75-99.

[SW] A.Suslin and M. Wodzicki, Excision in algebraic K-theory, Ann. of Math. 136(1), (1992), $51-122$.

[We] J.Weidner, Topological invariants for generalized operator algebras, Doctoral Thesis, University of Heidelberg, 1987. 